Methods: Transversal descriptive study. We included 13 patients diagnosed with SLE according to the criteria of the American College of Rheumatology (ACR) with positivity for antinuclear antibodies (ANA) and anti-DNA, 34 first-degree relatives and 50 healthy controls between the months of May 2016 and March 2017 None of the subjects evaluated received treatment with rituximab or belimumab. We analysed B-cells subsets (negative double, naïve B-cells, unswitched memory B-cells, switched memory B-cells) in all the subjects included in our study. The $95 \%$ confidence intervals were obtained for both the means and the percentage difference. The level of statistical significance was established at $p<0.05$. The data was analysed with IBM SPSS software Statistics 19 and EPIDAT 4.1.

Results: 47 subjects were analysed between relatives and patients, of which 33 ( $70.20 \%$ ) were women. 13 subjects (27.70\%) were diagnosed with lupus. $100 \%$ of those diagnosed with lupus were women. The mean $(\mathrm{X})$ and confidence intervals $(95 \% \mathrm{Cl})$ for the different subgroups (healthy subjects, subjects diagnosed with SLE, relatives of the first degree) is shown in table 1. In none of the subpopulations analysed in patients diagnosed with SLE in front of relatives of 1 st grade it has reached statistical significance. When analysing $B$ cells subsets of the three groups of subjects, we did find statistically significant differences between unswitched memory $B$ cells of healthy subjects and 1 st degree relatives (being lower in the group of healthy subjects) This finding has not been described in any previous study, although it should be noted that the sample before us is small.

Abstract OP0178 - Table 1

\begin{tabular}{lcccc}
\hline B-CELLS SUBSETS & & HEALTHY & SLE & RELATIVES \\
\hline Naïve (\%) & Mean & $70,4(95 \% \mathrm{Cl}:$ & $69,3(95 \% \mathrm{Cl}:$ & $65,8(95 \% \mathrm{Cl}$ \\
& $(\mathrm{Cl} 95 \%)$ & $68,2$ to 72,7$)$ & $62,2$ to 76,3$)$ & $60,4$ to 71,2$)$ \\
\hline Doubles Negative & Mean & $4,1(95 \% \mathrm{Cl}: 3,5$ & $6,2(95 \% \mathrm{Cl}: 4,8$ & $2,9(95 \% \mathrm{Cl}: 1,9$ \\
(DN) (\%) & $(\mathrm{Cl} 95 \%)$ & to 4,6$)$ & to 7,6$)$ & to 4,0$)$ \\
Unswitched (\%) & Mean & $9,6(95 \% \mathrm{Cl}: 8,2$ & $13,0(95 \% \mathrm{Cl}: 7,4$ & $17,2(95 \% \mathrm{Cl}:$ \\
& $(\mathrm{Cl} 95 \%)$ & to 11,1$)$ & to 18,7$)$ & $13,4$ to 21,1$)$ \\
Switched (\%) & Mean & $11,4(\mathrm{Cl})$ & $11,5(95 \% \mathrm{Cl}: 6,3$ & $14,0(95 \% \mathrm{Cl}$ \\
& $(\mathrm{Cl} 95 \%)$ & $=10,1-12,7)$ & to 16,7$)$ & $11,2$ to 16,8$)$ \\
\hline
\end{tabular}

Conclusions: There are quantitative differences between unswitched memory B-cells of healthy subjects and relatives of 1 st grade of SLE. More studies with a larger sample size are necessary to see the behaviour of the rest of B-type lymphocyte subpopulations

Disclosure of Interest: None declared

DOI: 10.1136/annrheumdis-2018-eular.7262

\section{OP0179 MOLECULAR DOCUMENTATION OF THE CLONAL EVOLUTION OF A DIFFUSE LARGE B-CELL LYMPHOMA OUT OF CLONALLY EXPANDED RHEUMATOID FACTOR-EXPRESSING B CELLS IN A SJÖGREN'S SYNDROME PATIENT}

R.J. Bende ${ }^{1}$, L.M. Slot ${ }^{1}$, T.A. Wormhoudt ${ }^{1}$, M.J. Kwakkenbos ${ }^{2}$, A.H. van Kampen ${ }^{3}$, A. Jongejan ${ }^{3}$, J.E. Guikema ${ }^{1}$, C.J. van Noesel ${ }^{1}{ }^{1}$ Pathology, Academic Medical Center, ${ }^{2}$ AIMM Therapeutics, AIMM Therapeutics; ${ }^{3}$ Clinical Epidemiology, Biostatistics and Bioinformatics, Academic Medical Center, Amsterdam, Netherlands

Background: Sjögren's syndrome (SS) is the autoimmune disease with the highest risk of lymphoma development. SS patients develop most frequently MALT lymphoma and to a lesser extent diffuse large B-cell lymphoma (DLBCL). Previously, we have shown that at least $40 \%$ of salivary gland MALT lymphomas express groups of near identical (also called stereotypic) B-cell receptors, which display in vitro mono-specific rheumatoid factor (RF) reactivity ${ }^{1}$. Recently, we have analysed the B-cell immunoglobulin heavy variable (IGHV) repertoire in 4 SS labial salivary glands. In 2 out of 4 salivary glands only one minor stereotypic RF B-cell clone was detected. Interestingly, in one salivary gland of patient SG22 a highly expanded stereotypic RF-expressing B-cell clone was present, which was also detected in peripheral blood. Twenty six months later, a clonally-related DLBCL was diagnosed ${ }^{2}$.

Objectives: To study the molecular alterations present in the RF-expressing $\mathrm{B}$ cell clones of salivary gland, peripheral blood and in the DLBCL of SS patient SG22.

Methods: From peripheral blood 4 RF-expressing immortalised B-cell clones with identical IGHV rearrangements were isolated. These 4 RF-clones were analysed by whole exome sequencing and the identified non-synonymous exome mutations were traced in the salivary gland and in the DLBCL, using targeted next generation sequencing.

Results: In total we identified 56 exome mutations in the 4 RF B-cell clones. Twelve non-synonymous mutations were shared between all 4 RF-clones, of which one was a missense mutation in CARD11, a well-known oncogenic mutation of DLBCL. In the salivary gland all shared 12 non-synonymous mutations were detected, whereas in the DLBCL only 4 of these mutations were detected.
Remarkably, the CARD11 mutation was not detected in the DLBCL. The exome mutations were analysed for typical characteristics of induction by the B-cell specific mutator AID. This revealed that the large majority of mutations $(80 \%)$ were (i) not located within $1,5 \mathrm{kB}$ of the transcription initiation site of the genes, (ii) did not concern C/G nucleotides in AID hotspots (WRC/GYW) and (iii) were not in genes expressed by B cells. This lack of evidence for AID involvement, suggests that the exome mutations of the RF-clones were due to replication errors, obtained during their clonal expansion. Interestingly, as compared to the RF-clones of peripheral blood, the DLBCL accumulated 44 extra somatic mutations together in IGHV and IGKV. As expected, these new IGHV/IGKV mutations showed the characteristics of AID involvement, indicating that in an ancestor RF-clone, AID activity was induced.

Conclusions: We have documented the clonal outgrowth and diversification of a RF-specific B-cell clone in a SS patient and the evolution of this clone into a DLBCL. Our analysis are indicative for AID activity in the lymphoma cells, represented by accumulation of IGHV/IGKV mutations. However, we have no evidence that AID is instrumental in the accumulation of the non-IG mutations in the RF clones from peripheral blood.

\section{REFERENCES:}

[1] Bende, et al. J. Exp. Med 2005;201:1229-1241.

[2] Bende, et al. Arthritis Rheumatol 2015;67:1074-1083.

Disclosure of Interest: None declared

DOI: 10.1136/annrheumdis-2018-eular.4401

\section{OP0180 TYPE I INTERFERON IS PRODUCED BY NON- HAEMATOPOIETIC TISSUE RESIDENT CELLS BUT NOT PDCS IN PRE-CLINICAL AUTOIMMUNITY AND SLE}

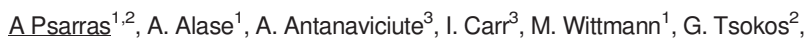

P. Emery ${ }^{1}$, E.M. Vital ${ }^{1} .{ }^{1}$ Leeds Institute of Rheumatic and Musculoskeletal Medicine, University of Leeds, Leeds, UK; ${ }^{2}$ Beth Israel Deaconess Medical Center, Harvard Medical School, Boston, USA; ${ }^{3}$ Leeds Institute for Data Analytics, University of Leeds, Leeds, UK

Background: Systemic Lupus Erythematosus (SLE) is characterised by persistently high type I interferon (IFN) activity. Plasmacytoid dendritic cells ( $p D C s$ ) pro duce large amounts of IFNs in viral infection, although these immunogenic properties are usually strictly regulated. In vitro, pDCs are responsive to nucleic acids and they have therefore been postulated to be the main source of type IFNs in SLE. However, their function is not fully established in human SLE.

Objectives: To investigate the dysregulated IFN axis in patients with pre-clinical autoimmunity and SLE.

Methods: Patients with SLE who met 2012 ACR/SLICC criteria were recruited. We also recruited healthy controls $(\mathrm{HC})$ and therapy-naïve individuals presenting with ANA and 1-2 clinical symptoms, but not meeting ACR/SLICC criteria, of whom $17 \%$ progressed to SLE (At-Risk). IFN activity was evaluated by measuring a score of IFN-responsive genes in the PBMCs using TaqMan. pDCs were immunophenotyped as well as studied in vitro for production of proinflammatory cytokines and induction of $\mathrm{T}$ cell responses using flow cytometry. pDCs were sorted and sequenced using high-sensitive RNA sequencing. IFN expression was visualised in skin biopsies using in situ hybridisation. Keratinocytes were isolated from fresh skin biopsies and cultured in vitro; IFN production was measured by qPCR and ELISA.

Results: Most of the SLE and At-Risk patients had increased IFN activity, which correlated with disease activity and clinical features. In contrast, circulating pDCs were decreased in both SLE and At-Risk patients and their numbers did not correlate with any clinical features or IFN status. In vitro stimulation revealed that $\mathrm{pDCs}$ from SLE and At-Risk patients could not produce IFN- $\alpha$ and TNF- $\alpha$ upon stimulation with TLR9 or TLR7 agonists. In addition, they induced significantly less T cell activation and proliferation compared to $\mathrm{HC}$ pDCs. RNA-seq data analysis showed an upregulation of IFN-responsive genes in most of the SLE and At-Risk pDCs but not transcripts of any IFN subtypes. Other upregulated pathways were related to immune regulation and senescence. Phenotypically, SLE pDCs were characterised by upregulation of regulatory receptors and increased telomeric erosion. In situ hybridization revealed high IFN expression in the epidermis but not in lymphocyte-infiltrating areas of lesional biopsies from SLE patients. High expression of IFN was also observed in epidermis of At-Risk individuals without any signs of cutaneous inflammation. In vitro stimulation of freshly isolated keratinocytes also showed a notable increase in IFN production.

Conclusions: In SLE, non-haematopoietic tissue resident cells are a dominant source of IFN and this is present prior to clinically overt disease. Meanwhile, the professional IFN-producing pDCs have lost their immunogenic properties. These findings suggest an important role for tissue resident cells in autoimmunity and may facilitate novel therapeutic interventions.

Disclosure of Interest: None declared 